\title{
Resurrection of the Island Rule: Human-Driven Extinctions Have Obscured a Basic Evolutionary Pattern
}

\author{
Søren Faurby ${ }^{1,2, *}$ and Jens-Christian Svenning ${ }^{2}$
}

1. Department of Biogeography and Global Change, Museo Nacional de Ciencias Naturales, Consejo Superior de Investigaciones Científicas, Calle José Gutiérrez Abascal 2, Madrid 28006, Spain; 2. Section for Ecoinformatics and Biodiversity, Department of Bioscience, Aarhus University, Ny Munkegade 114, DK-8000 Aarhus C, Denmark

Submitted June 8, 2015; Accepted January 29, 2016; Electronically published April 12, 2016

Online enhancements: appendix, zip file. Dryad data: http://dx.doi.org/10.5061/dryad.2sq1r.

\begin{abstract}
Islands are or have been occupied by unusual species, such as dwarf proboscideans and giant rodents. The discussion of the classical but controversial island rule-which states that mammalian body sizes converge on intermediate sizes on islands-has been stimulated by these unusual species. In this study, we use an unprecedented global data set of the distributions and body sizes of late Quaternary mammal species and a novel analytical method to analyze body size evolution on islands. The analyses produced strong support for the island rule. Islands have suffered massive human-driven losses of species, and we found that the support for the island rule was substantially stronger when the many late Quaternary extinct species were also considered (particularly the tendency for dwarfing in large taxa). The decisive support for the island rule in this study confirms that evolution plays out in a markedly different way on islands and that human impact may obscure even fundamental evolutionary patterns.
\end{abstract}

Keywords: Anthropocene, body size, evolution, islands, mammals.

\section{Introduction}

Before the arrival of humans, many oceanic islands housed bizarre mammal faunas. Dwarf proboscideans used to occur on Mediterranean islands, the Channel Islands in California, and the island of Timor in Southeast Asia, but all are extinct (Faurby and Svenning 2015). Similarly, giant rats were frequent on islands but with only a few species still extant (Faurby and Svenning 2015) and, in some cases, with much reduced range, for example, the Malagassy giant rat (Hypogeomys antimena; Burney et al. 2008). In addition to these clades with numerous deviant island forms, many other clades also had a single or a few odd-sized island species, for example, the extinct dwarf hippos of Crete and Madagascar

* Corresponding author; e-mail: faurby@mncn.csic.es.

Am. Nat. 2016. Vol. 187, pp. 812-820. () 2016 by The University of Chicago. 0003-0147/2016/18706-56323\$15.00. All rights reserved. DOI: $10.1086 / 686268$ and the extinct Sardinian giant pika (Stuenes 1989; Angelone et al. 2008).

These bizarre island mammals have stimulated the proposal for the island rule, which states that mammalian body sizes converge on intermediate sizes on islands (Van Valen 1973). However, the island rule has been intensely debated in recent years and is viewed alternately as a near universal rule (Lomolino et al. 2012) or a sample or publication artifact (Meiri et al. 2008; Raia et al. 2010), with intermediate positions also argued (Welch 2009). Both opponents and proponents of the island rule acknowledge that islands harbor an apparent abundance of species with extreme body sizes, that is, giant forms of small species and dwarf forms of large species (Meiri et al. 2008; Lomolino et al. 2012). However, the two schools have strongly debated whether the island rule represents a general evolutionary pattern, the idiosyncratic changes in individual lineages, or even the human tendency to see patterns in all data sets (Van Valen 1973; Meiri et al. 2008; Raia et al. 2010; Lomolino et al. 2012).

Critics of the island rule argue two main points, both of which we overcome in this study. The first point concerns sampling bias. The studies that support the island rule have generally been meta-analyses of published comparisons between the mainland and island populations of the same species (Van Valen 1973; Lomolino et al. 2012). As discussed for the related Bergmann's rule (Meiri et al. 2004), these studies may be a nonrandom subset of all populations, and therefore a significant pattern-matching expectation may be generated by a reporting bias. In this study, we removed the possibility for such sampling bias by generating and analyzing a database that contained the body sizes for approximately $99 \%$ of all extant and recently extinct species of mammals (see "Material and Methods").

The second critique of the basis for the island rule is related to phylogenetic nonindependence. Previous studies have showed diminished support for the rule after accounting for phylogeny in intraspecific analyses (Meiri et al. 2008; 
McClain et al. 2013). This problem is a form of pseudoreplication, inflating estimates of precision and thereby potentially generating type I errors. The magnitude (or existence) of this problem, however, depends on what model is used as the null model. As also pointed out by Welch (2009), the classical studies, which compared only sister lineages (e.g., Lomolino 1985), are compatible with body sizes that evolved via simple models, such as Brownian motion (Felsenstein 1985) or Ornstein-Uhlenbeck (OU) models (Hansen 1997). Studies that have analyzed ratios between sizes of island and mainland mammals in a phylogenetic context (e.g., Meiri et al. 2008) might also be compatible with such models, if one assumes an identical age for all island populations (which is clearly wrong, since island endemics have ages ranging from Paleogene to Holocene [Anderson and Handley 2001; Dewar and Richard 2012]). They could also be valid if one is willing to assume no effect of the age of an island clade on the ratio between the size of the island and mainland clade. This would, however, require that changes in body size occurred only at speciation and not during the later life of a species, which numerous studies have also shown to be wrong (e.g., Meachen and Samuels 2012).

Alternatively, if the rate of evolutionary change is a function of traits-which are also evolving, for example, via the correlation between generation length and evolutionary rate (Welch et al. 2008; Thomas et al. 2010) - phylogenetic nonindependence is a problem for studies that do not integrate phylogeny. Such a correlation is not a problem for studies that incorporate phylogeny or that focus on the ratios between the island and mainland species, but such studies again require an identical age of all island populations or that the island populations have reached a new equilibrium size. Imagine, for example, an analysis that contained two sets of rodent mainland/island sister pairs with short generation times (and therefore potentially fast evolutionary rates) and two sets of elephant mainland/ island species pairs with longer generation times (and therefore potentially lower evolutionary rates). If the rate is evolving over time, the comparisons of the magnitude of change between the species pairs will need phylogenetic correction because larger relative differences between the mice species pairs than the elephant species pairs could be a null expectation. Irrespective of whether the rate is evolving, however, the null expectations would remain a $50 \%$ decrease in size in both mice and elephants, as long as neither clade is undergoing a consistent change in body size.

To solve the potential problem of phylogenetic nonindependence without requiring an identical age of all island populations or that the island populations have previously reached a new equilibrium size, we restricted the analyses to focus only on the directionality and not on the magni- tude of change (see "Material and Methods"). We stress that this restriction does not imply that body size evolution departs from the expectation under a Brownian process, which often may be a good approximation (Blomberg et al. 2003; although see Uyeda et al. 2011), but rather that our analysis (explained below) did not assume Brownian motion to be true. Moreover, the analysis was almost independent of the assumed model of body size evolution.

In addition to the potential problems with the studies that support the island rule, the primary interspecific study that dismisses the island rule (Raia et al. 2010) also has potential problems. The study used a somewhat incomplete body size database (Smith et al. 2003) and a partially outdated phylogeny (Bininda-Emonds et al. 2007). Raia et al. (2010) also included bats, whereas the classic studies that support the island rule focused on nonflying mammals (Lomolino 1985) or analyzed bats separately (Lomolino 2005). If supported, the island rule is likely a consequence of island isolation, and the substantially lower levels of endemism in bats than in nonflying mammals (Weyeneth et al. 2011) indicates that the island bat fauna is less isolated compared with nonflying mammalian fauna. Thus, the island rule would be expected to establish a weaker pattern for bats than for nonbats.

Bats are also illustrative of another suggested problem of the island rule but one we find highly unlikely to be important. Meiri et al. (2008) discuss bats as a group that does not obey the island rule and argue that this supports that the rule is not valid but instead is consistent with some clades growing and others shrinking in body size on islands independently of their mainland body size, with the apparent rule simply a consequence of pseudoreplication. However, no mechanistic null model showing directional size decreases in some clades and increases in others is suggested. We think that the problem is more likely to be unmeasured parameters, which may or may not be phylogenetically clustered. The problem is therefore likely better addressed by incorporating the relevant ecological factors than incorporating phylogeny. In other words, if all bats disobey the island rule, it may not be because they are related but because they all are capable of flight. Since flight has evolved only once in mammals, the genetic distance between mammals and the ability to fly are correlated. An analysis on the island rule focusing on only body size and not phylogeny may therefore not be as appropriate as the same analysis incorporating phylogeny in such cases, but none of these may be as appropriate as an analysis using body size and the capacity for flight.

In this article, we reanalyzed the validity of the island rule in an interspecific context using a novel, near-complete body size database and a recent mammalian phylogeny (Faurby and Svenning 2015) focusing solely on the directionality and not on the magnitude of body size changes in island lineages. To determine the potential importance of the factors 
responsible for the apparent lack of support for the island rule in the earlier studies that integrated phylogenetic relationships between species, we estimated the effects of including or excluding bats and extinct species as well as different definitions of islands.

\section{Material and Methods \\ Data Generation}

For all analyses, we used the taxonomy and genetic relationships of a recent mammalian phylogeny, which included all species with dated occurrences within the past 130,000 years but lumping likely chronospecies (Faurby and Svenning 2015). Notably, most extant mammal species existed throughout this period and therefore coexisted with the extinct species, and there is increasing evidence that Homo sapiens was the primary cause of these extinctions on mainlands (Sandom et al. 2014) as well as islands (Turvey and Fritz 2011).

We generated a new body size database, which included almost all known late Quaternary mammal species (5,673 of 5,747 species; of the 74 species without data, eight represented extinct known but undescribed species). The new database was partly based on an older database (Smith et al. 2003) but was heavily modified. The information for 3,629 of the 5,673 species was used from the older database, but our complete database contained information from a total of 709 separate data sources (644 articles published in 146 separate journals, 55 books, eight web resources, and personal information from two experts; the complete database is deposited in the Dryad Digital Repository, http:// dx.doi:10.5061/dryad.2sq1r [Faurby and Svenning 2016], and is also available in the supplementary data [available online], in addition to information on which islands all island endemic species are found). For the species for which the weight data were not available, the weights were generally estimated with the assumption of strict isometries for related similar-sized species. Isometry was generally assumed for forearm length in bats and body length (excluding tail) for the remaining species, but other measures were also used occasionally.

We scored island endemicity as a binary character and defined island endemics on the basis of three definitions. The first definition of island endemics (classical definition) considers any species as an island endemic that is endemic to oceanic islands at the current sea level. The second definition (strict definition) considers species as island endemics only if they are restricted to islands that have not been not connected to a continent during Pleistocene glaciations (i.e., islands for which the deepest water level between the island and a continent is more than $110 \mathrm{~m}$ deep). The third definition (semistrict definition) considers species as island endem- ics if they are endemic to either islands not connected to the mainland during Pleistocene glaciations or small landbridge islands $\left(<1,000 \mathrm{~km}^{2}\right)$. Irrespective of the definition, we did not score species as island endemics if they are known from the mainland within the Holocene. Hence, the Tasmanian devil (Sarcophilus harrisii) and the Tasmanian tiger (Thylacinus cynocephalus) were not considered island endemics under any of our definitions, since they both occurred on mainland Australia until the mid-Holocene (Johnson and Wroe 2003).

The intent of the strict definition is to score species as island endemics only if they have been restricted to islands for the majority of their evolutionary history rather than just at high interglacial sea levels (such as during the Holocene). For the few species that have evolved by rapid speciation since the Last Ice Age on land-bridge islands (e.g., Anderson and Hadley 2001), this definition is overly restrictive because they are excluded despite having been island endemics for their entire evolutionary history. The semistrict definition was therefore used to include all island species originating after the last ice age as island endemics, even if they occur on land-bridge islands. We do not know the precise age of all species and therefore chose a size threshold for island size, since rapid speciation likely has required a small population size and therefore a limited area. We acknowledge that no strong arguments exist for setting this threshold to exactly $1,000 \mathrm{~km}^{2}$. However, our threshold means that the largest island with a strong candidate for such recent speciation (the endemic agouti Dasyprocta coibae on the land-bridge island Coiba [503 $\left.\mathrm{km}^{2}\right]$ ) is considered an island endemic. On the other hand, the colobus monkey Procolobus kirkii from the somewhat larger land-bridge island Zanzibar $\left(1,658 \mathrm{~km}^{2}\right)$ - which has been analyzed genetically and found to be Middle Pleistocene in age (Ting 2008) and thus has existed also when Zanzibar was part of the African mainland during glaciations-is not considered an island endemic by this definition.

\section{Analyses}

The phylogeny used in this study consisted of 1,000 separate fully bifurcating, random trees from a posterior distribution of trees that represented the phylogenetic uncertainty from Faurby and Svenning (2015). For these analyses, we focused on the probability of decrease in body size after the colonization of islands. As noted later, we excluded all cases with no change in body size between island and mainland species, meaning that the probability of size increase is equal to 1 minus this probability. For the island rule to be true, we would expect the probability of size decrease to be lower than 0.5 for small species and higher than 0.5 for large species. The larger the effect of body size is on this probability, the stronger the support for the island rule. We analyzed the 
probability of size change as a function of body mass on the basis of a set of polynomials. The polynomials were used to allow flexible responses between the dependent and independent variables, reducing the need for assuming a certain functional response. In this regard, we note that support for the island rule can only ambiguously be inferred if the relationship between body size and the probability of size decrease is monotonic (which it is for all our analyses). We reported our results on the basis of the probability of size decrease weighted across models and phylogenetic trees. It has recently been stressed that while it is important to incorporate estimates from multiple models, parameters cannot simply be averaged when there is multicollinearity among predictors (Cade 2015). Because the different terms in polynomials will necessarily be correlated, we therefore averaged the predicted probability of size decrease across the models rather than averaging the individual parameter values.

Separate analyses were initially performed for each of the 1,000 trees, after which the results from each tree were combined. For each island endemic species, we found the largest clade that contained only island endemics $\left(C_{\text {island }}\right)$ and the smallest clade that contained both island endemics and nonendemics, and we removed all members of $C_{\text {island }}$ from this clade (hereafter $C_{\text {mainland }}$ ). We then estimated ancestral $\log _{10}$ (weight) for all $C_{\text {island }}$ and $C_{\text {mainland, assuming }}$ Brownian motion (hereafter size mainland $_{\text {and }}$ and island $_{\text {) }}$. With the removal of the island endemics for the calculation of $C_{\text {mainland, }}$, we allowed body size evolution to differ between island and mainland clades but did not enforce such differences. Following this procedure, we sampled all the island endemic species in random order, listed all members of $C_{\text {island }}$, and, if the sampled species was not a member of the $C_{\text {island }}$ of any previously sampled species, noted the size of the size $_{\text {mainland }}$ and whether this size was smaller or larger than size $_{\text {island }}$. Therefore, our end products were a vector of ancestral mainland weights for independent island invasions and a corresponding vector with binary information on whether the island species were smaller than their mainland ancestors. To reduce the effects of measurement errors on weight, we discarded all island invasions for which the difference in weight between size mainland $_{\text {and }}$ size island $_{\text {was }}$ smaller than $10 \%$ from further analyses. Supplementary analyses were performed using $0 \%, 5 \%, 15 \%$, and $20 \%$ weight difference thresholds, but the results changed very little, although with a tendency for a weaker island rule for the $0 \%$ threshold, possibly a consequence of increased noise in the data (see supplementary figs. S1-S5). Regarding these thresholds, we furthermore caution that for all thresholds above $0 \%$, there is a minor risk to generate a small bias if, as we have suggested in the introduction, there is phylogenetic signal in the speed of body size evolution, since the slowest evolving clades will be more likely to be removed from the analysis.
We believe, however, that this potential bias will be smaller than the noise introduced by body size estimation errors if the magnitude of body size change was analyzed instead; hence, analyses focused purely on the existence (with a small threshold) and directionality of a body size change should be the best option.

We then fitted 0 to the fourth-degree polynomial models of the probability of size decrease as a function of the size mainland using logistic regression $\left(M_{0_{\text {treci }}}, M_{1_{\text {treci }}}, M_{2_{\text {trece }}}, M_{3_{\text {trec }}}, M_{4_{\text {treci }}}\right)$ and calculated their respective Akaike information criterion (AIC) weights $\left(w_{0_{\text {treci }}}, w_{1_{\text {trec }}}, w_{2_{\text {trece }}}, w_{3_{\text {trece }}}, w_{4_{\text {treci }}}\right)$. For all the potential values of size mainland $_{\text {metween }}$ betwe and 6.0 (i.e., untransformed weights between $1 \mathrm{~g}$ and 1 ton) in steps of 0.1 for all models, we then calculated the means and the variances,

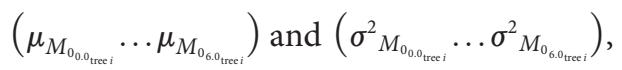

respectively, for the untransformed fitted values for all five models.

The results were thereafter combined for all five models for each potential value of $C_{\text {mainland }}$ as a mixture of the normal distributions from the five models, with the weight of each model equal to the AIC weight. Therefore, the combined result was that the predicted effect for any body size (k) would be in the distribution

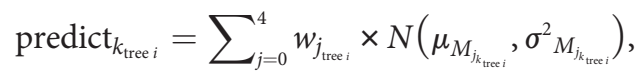

where $i$ is one of the 1,000 trees and $j$ represents the order of the polynomial ranging from 0 to 4 . Following this procedure, the results were combined for all trees as

$$
\text { combined }_{k}=\sum_{i=1}^{1,000} \text { predict }_{k_{\text {tre } i}} .
$$

Finally, the median and several quantiles for the combined ${ }_{k}$ were transformed into probabilities.

We tested the effect of the definition of an island endemic, the potential effect of the anthropogenic extinctions to bias the results, and the effect of including bats in the analysis. This analysis was performed separately for each of the 12 combinations of the three definitions of island endemics (classical, semistrict, strict), the exclusion or inclusion of bats, and the exclusion or inclusion of extinct species.

For all analyses, in this article we assume a completely genetic basis for body size. We acknowledge this to be potentially problematic since plasticity rather than genetics often drives changes in average body sizes within populations, at least over short timescales (see discussion in Boutin and Lane 2014), and plasticity therefore likely also plays a role in comparisons between populations or species. We believe 
that this potential bias is unlikely to be of sufficient magnitude to generate wrong conclusions and note that as long as the plastic effects are relatively small, they should influence only comparisons where mainland and island species are essentially of the same size. Plasticity would therefore be expected to be a larger problem for the comparisons with smaller thresholds for the difference between mainland and island clades to count as a size change, but our results remain stable across the broad range of tested thresholds (figs. S1-S5). We address a number of other potential problems (and why none of them are likely to be problematic for our analysis) in the appendix, available online.

All analyses were performed in R (ver. 3.0.2; R Development Core Team 2013), using functions from the libraries ape (Paradis et al. 2004), phylobase (R Hackathon et al. 2014), and qpcR (Spiess 2014).

\section{Results}

Strong support for the island rule was provided when bats were excluded from the analysis, while it was only weakly supported when the bats were included. Among the 12 combinations of island-type definitions and included species, the strongest support for the island rule (measured as the difference between the predicted probability for size increase species for species with a size of $1 \mathrm{~g}$ and $1 \mathrm{ton}$ ) was with the strict island definition and exclusion of bats but inclusion of extinct species (table 1; figs. 1, S1-S5). The inclusion of bats in the analysis consistently led to markedly lower support for the island rule, because the addition of the bats removed or at least reduced the tendency for small mammals to increase in size on islands. The inclusion of the extinct species and the application of the strict or semistrict island definitions provided stronger support for the island rule, but only when bats were excluded.

\section{Discussion}

The validity of the island rule was clearly supported by our analyses. We suggest that part of the explanation for the lack of evidence for the island rule in the earlier interspecific study (Raia et al.2010) is not incorporation of the phylogeny, as the authors suggested. We consider it more likely that it was caused by their choice to disregard the ecological difference between flying and nonflying mammals and, to a lesser extent, their choice of definition of island endemics and the incomplete inclusion of historically extant species (i.e., species that occurred within the Late Pleistocene or Holocene). In this regard, we do not state explicitly that the models that excluded bats in our analysis provided a better fit to the data than the models that included bats; rather, we state that the estimated effect of body size (i.e., the island rule) is substantially stronger in the models that excluded bats.

Since our results clearly showed that the island rule is a valid phenomenon, the question becomes what factors could be driving it, since numerous drivers have been suggested (see, e.g., discussion in Lomolino et al. 2012). Most of these drivers focus on what happens after the colonization, but one potential driver-immigrant selection-is noteworthy for postulating that the driver for gigantism in small species instead is that the largest individuals are most likely to survive the transport to the islands. Immigrant selection could be important at the relatively short timescales reflected in intraspecific analyses, but considering the evolutionary rates over small to medium timescales (Gingerich 2001), any effect of immigrant selection must be expected to have disappeared in interspecific analyses. Stabilizing selection should move the average body size of the island species back to the average size of the mainland population within this time frame, unless the selective forces governing body size differ between mainland and island (for a similar argument regarding body size evolu-

Table 1: Estimated strength of the island rule under the 12 different analyzed scenarios

\begin{tabular}{lcccc}
\hline Island-endemic definition & Including extinct species & Including bats & Difference $^{\mathrm{a}}$ & $2.5 \%-97.5 \%$ quantiles \\
\hline Classical & No & Yes & .621 & $-.137-.988)$ \\
Classical & No & No & .733 & $.038-.972$ \\
Classical & Yes & Yes & .602 & $.229-.940$ \\
Classical & Yes & No & .758 & $.351-.994$ \\
Semistrict & No & Yes & .535 & $-.262-.952$ \\
Semistrict & No & No & .841 & $-.009-.986$ \\
Semistrict & Yes & Yes & .540 & $-.019-.898$ \\
Semistrict & Yes & No & .876 & $.021-1.000$ \\
Strict & No & Yes & .556 & $-.230-.972$ \\
Strict & No & No & .863 & $-.033-.999$ \\
Strict & Yes & Yes & .572 & $-.020-.947$ \\
Strict & Yes & No & .904 & $.019-1.000$ \\
\hline
\end{tabular}

${ }^{a}$ Difference between the predicted probability of size increase for species of $1 \mathrm{~g}$ and 1 ton. 

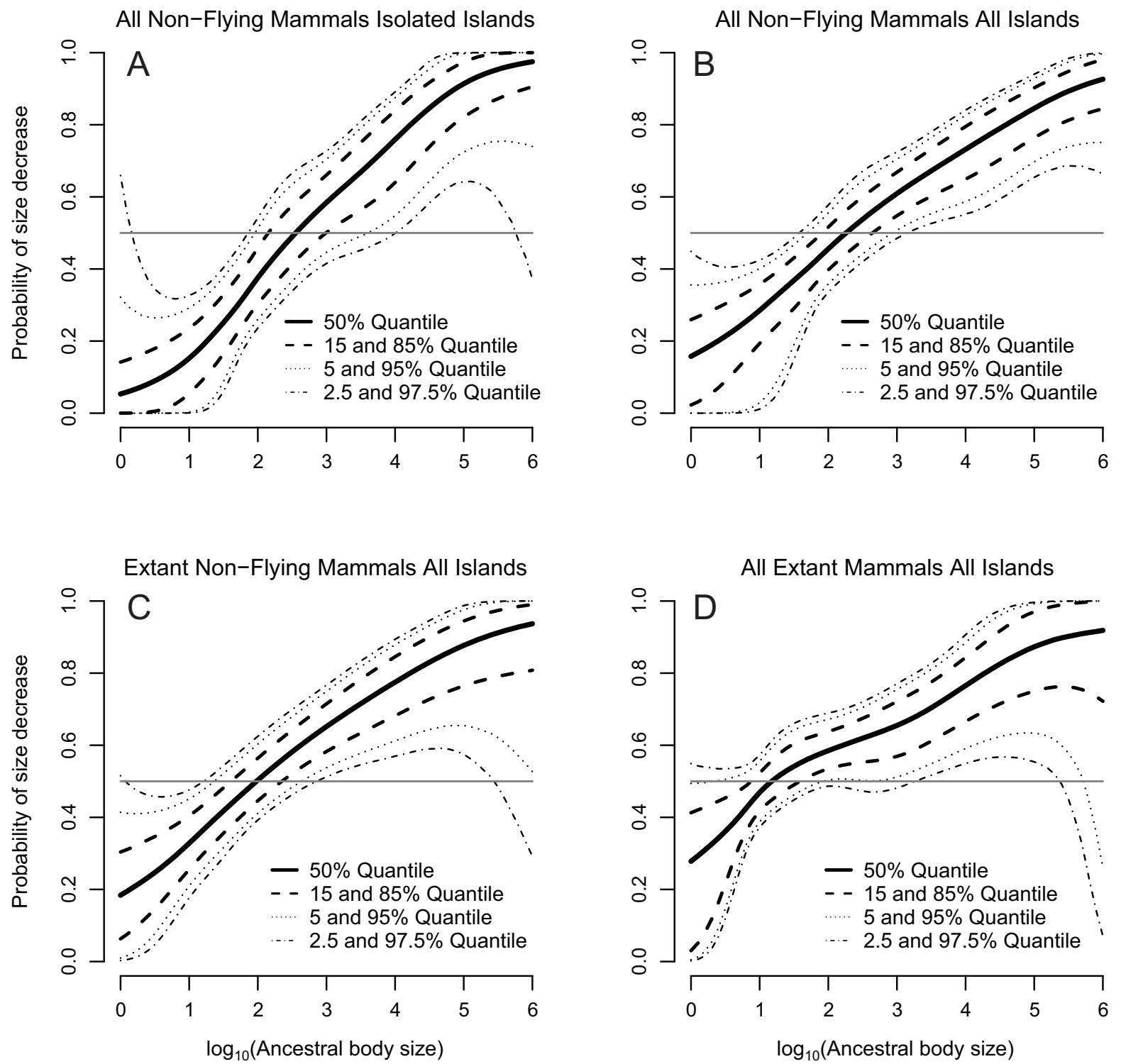

Figure 1: Relationships between ancestral body size and directionality of evolutionary size change after island invasion. The estimated probability of a size decrease as a function of the ancestral body size of the island invading clade. Solid black line shows the median of the distribution of potential predicted values, whereas the three stippled lines show the $2.5 \% / 97.5 \%, 5 \% / 95 \%$, and $15 \% / 85 \%$ quantiles. Because the response variable is binary, values below the horizontal line indicate that a clade is most likely to increase in size, and values above the horizontal line indicate that a clade is most likely to decrease in size. A shows the relationship for nonflying mammals, including both extinct and extant species, for isolated islands. $D$ shows the relationship for both flying and nonflying mammals but only for extant species and using all islands. The three differences between $B-D$ are changed one by one; $B-D$ use all the islands, $C$ and $D$ analyze only extant species, and $D$ analyzes all mammals without restricting the analysis to nonflying species.

tion in island tortoises, see Jaffe et al. 2011); our results therefore suggest that another driver than immigrant selection is needed.

We suspect that the primary driver behind the island rule is ecological release. Body sizes of mainland species are likely to be at least partly driven by competition and predation (Brown and Wilson 1956). If one of the reasons for large body size in the largest species, such as elephants, is reduced susceptibility to predation, it would explain why elephants generally dwarf in islands, that is, given the absence of large carnivores, which cannot normally maintain minimum viable population sizes on islands because of the small area available. For small species, on the other hand, lower predation could reduce selection for early reproduction, leading to larger adult size. Competition may likewise be important if within a given guild there is a more or less 
bell-shaped response between body size and the part of the resource that can be utilized. This can be illustrated by looking at a couple of hypothetical insectivorous shrews. If the shrew gets too small, the largest insects may get increasingly difficult to catch, while if the shrew gets too big, the smallest insects may no longer be energetically viable to catch. Hence, an intermediate size should be optimal if only one shrew species occurs in a habitat. If two shrew species are present, character displacement may on the other hand be beneficial to reduce competition, and the development of one large and one small species will maximize the available resources for each species.

The pattern of mammalian body sizes on islands has sometimes instead been interpreted in the context of differential extinction since data suggest a lower extinction rate of intermediate-sized species since intermediate-sized species often have higher density and therefore can maintain a stable population on small islands (Marquet and Taper 1998). One could therefore hypothesize that body sizes of island species develop in random directions and that the species evolving similar to the expectations of the island rule are the ones surviving. We cannot rule out that this plays a role, but note that a lower extinction for intermediate-sized species could also be driven by less resource availability for extreme-sized animals, as described above.

The effects of including or excluding land-bridge islands and bats into the analysis provided support for ecological release as the main driver of island rule. In this case, the island rule should become realized only for taxa on islands with reduced numbers of predators or competitor species. Therefore, the island rule should not apply or should be much less applicable to land-bridge islands, as was indeed the case in our analyses, because these have been part of the continental mainland during the last glaciation, in addition to many earlier periods during the Pleistocene. The species on the land-bridge islands would have experienced similar faunas as those on mainlands for a large part of their evolutionary history; therefore, these species would not have experienced ecological release or only a relatively brief release (during the Holocene), which is likely insufficient for substantial changes in body size to develop. Similarly, island bats are not likely to have experienced significant ecological release on islands because the primary predators of bats are birds such as raptors and owls (Rydell and Speakman 1995). These birds are typically strong fliers, and therefore even long isolated islands tend to harbor welldeveloped predatory bird faunas. Thus, for the native bat fauna on islands, predator release would be limited or would not occur. Again, this is consistent with our finding that including bats in the analyses substantially reduced support for the island rule.

With the arrival of humans, island faunas suffered severe extinctions. Our data set included 589 nonflying and 223 flying island endemics based on the strict island definition, with overall late Quaternary extinction rates of $20 \%$ and $4 \%$, respectively (the corresponding number for all the islands was 916 nonflying and 323 flying species with extinction rates of $13 \%$ and $3 \%$, respectively; see supplementary data). These extinctions are often tightly linked to human arrival and to evidence of human hunting or other anthropogenic factors (Turvey and Fritz 2011). Based on our analysis, the inclusion of the extinct species strongly increased the support for the island rule. The incorporation of the extinct island species was previously advocated for ecological studies (Griffiths et al. 2009; Hansen and Galetti 2009), but our results highlighted the necessity to also include these species in evolutionary studies. The recent human-driven extinctions most likely have obscured signals related to the long-term evolutionary responses to island environments, for example, the elimination of the most specialized island lineages (Lomolino et al. 2013). In this regard, we note that since both dwarfing and gigantism may be an evolutionary consequence of predator release, as discussed above, the species with largest changes in body size on islands would be expected to be the most sensitive to predation by humans or our commensal animals.

In this study, the decisive support for the island rule highlights that the function of island ecosystems is fundamentally different from that of mainland systems (cf. Millen 2006) and that these differences drive divergent evolutionary dynamics on islands and the mainland. Notably, our results were consistent with a weakening of ecological interactions on islands, causing body sizes to shift to intermediate biomasses, irrespective of the ancestral body size or the phylogenetic lineage. Conversely, the strong support for the island rule also implies that much of the large variation in body sizes and the repeated evolution of similar maximum body sizes in mainland systems (Smith et al. 2010) must be consequences of intense ecological interactions in these settings.

\section{Acknowledgments}

S.F. was supported by the Danish Natural Science Research Council (4090-00227). J.-C.S. was supported by the European Research Council (ERC-2012-StG-310886-HISTFUNC). We greatly appreciate input on earlier versions of the manuscripts from the reviewers P. A. Marquet and A. Phillimore and from S. Kalisz and A. J. Kerkhoff. In particular, we thank A. Phillimore for his suggestion for how best to estimate the strength of the potential analytical problem we discuss in the appendix.

\section{Literature Cited}

Anderson, R. P., and C. O. Handley. 2001. A new species of threetoed sloth (Mammalia: Xenarthra) from Panama, with a review of 
the genus Bradypus. Proceedings of the Biological Society of Washington 114:1-33.

Angelone, C., C. Tuveri, M. Arca, N. L. Martinez, and T. Kotsabis. 2008. Evolution of Prolagus sardus (Ochotonidae, Lagomorpha) in the Quaternary of Sardinia Island (Italy). Quaternary International 182:109-115.

Bininda-Emonds, O. R. P., M. Cardillo, K. E. Jones, R. D. E. MacPhee, R. M. D. Beck, R. Grenyer, S. A. Price, R. A. Vos, J. L. Gittleman, and A. Purvis. 2007. The delayed rise of present-day mammals. Nature 446:507-512.

Blomberg, S. P., T. G. Garland, and A. R. Ives. 2003. Testing for phylogenetic signal in comparative data: behavioral traits are more labile. Evolution 57:717-745.

Boutin, S., and J. E. Lane. 2014. Climate change and mammals: evolutionary versus plastic responses. Evolutionary Applications 7:2941.

Brown, W. L., and E. O. Wilson. 1956. Character displacement. Systematic Zoology 5:49-64.

Burney, D. A., N. Vasey, L. R. Godfrey, W. L. Jungers, Ramilisonina, M. F. Ramarolahy, and L. L. Raharivony. 2008. New findings at Andrahomana Cave, Southeastern Madagascar. Journal of Cave and Karst Studies 70:13-24.

Cade, B. S. 2015. Model averaging and muddled multimodel inferences. Ecology 96:2370-2382.

Dewar, R. E., and A. F. Richard. 2012. Madagascar: a history of arrivals, what happened, and will happen next. Annual Review of Anthropology 41:495-517.

Faurby, S., and J.-C. Svenning. 2015. A species-level estimate of the phylogeny for all extant and Late Quaternary extinct mammals using a novel hierarchical Bayesian approach. Molecular Phylogenetics and Evolution 84:14-26.

2016. Data from: Resurrection of the island rule: human-driven extinctions have obscured a basic evolutionary pattern. American Naturalist, Dryad Digital Repository, http://dx.doi:10.5061/dryad.2sq1r.

Felsenstein, J. 1985. Phylogenies and the comparative method. American Naturalist 125:1-15.

Genz, A., F. Bretz, T. Miwa, X. Mi, F. Leisch, F. Scheipl, and T. Hothorn. 2015. mvtnorm: multivariate normal and $\mathrm{t}$ distributions. $\mathrm{R}$ package, version 1.0-3. http://CRAN.R-project.org/package $=$ mvtnorm.

Gingerich, P. D. 2001. Rates of evolution on the time scale of the evolutionary process. Genetica 112/113:127-144.

Griffiths, C. J., D. M. Hansen, C. G. Jones, N. Zuël, and S. Harris. 2009. Resurrecting extinct interactions with extant substitutes. Current Biology 21:762-765.

Hansen, D. M., and M. Galetti. 2009. The forgotten megafauna. Science 324:42-43.

Hansen, T. F. 1997. Selection and the comparative analysis of adaptation. Evolution 51:1341-1351.

Jaffe, A. L., G. J. Slater, and M. E. Alfaro. 2011. The evolution of island gigantism and body size variation in tortoises and turtles. Biology Letters 7:558-561.

Johnson, C. N., and S. Wroe. 2003. Causes of extinction of vertebrates during the Holocene of mainland Australia: arrival of the dingo, or human impact? Holocene 6:941-948.

Lomolino, M. V. 1985. Body size of mammals on islands: the island rule re-examined. American Naturalist 125:310-316.

- 2005. Body size evolution in insular vertebrates: generality of the island rule. Journal of Biogeography 32:1683-1699.

Lomolino, M. V., D. F. Sax, A. R. Palombo, and A. A. van der Geer. 2012. Of mice and mammoths: evaluations of causal explanations for body size evolution in insular mammals. ournal of Biogeography 39:842-854.

Lomolino, M. V., A. A. van der Geer, G. A. Lyras, M. R. Palombo, D. F. Sax, and R. S. Rozzi. 2013. Of mice and mammoths: generality and antiquity of the island rule. Journal of Biogeography 40:1427-1439.

Marquet, P. A., and M. L. Taper. 1998. On size and area: patterns of mammalian body size extremes across landmasses. Evolutionary Ecology 12:127-139.

McClain, C. R., P. A. P. Durst, A. G. Boyer, and C. D. Francis. 2013. Unravelling the determinants of insular body size shifts. Biology Letters 9:20120989.

Meachen, J. A., and J. X. Samuels. 2012. Evolution in coyotes (Canis latrans) in response to the megafaunal extinctions. Proceedings of the National Academy of Sciences of the USA 108:15908-15913.

Meiri, S., N. Cooper, and A. Purvis. 2008. The island rule: made to be broken? Proceedings of the Royal Society B: Biological Sciences 275:141-148

Meiri, S., T. Dayan, and D. Simberloff. 2004. Carnivores, biases and Bergmann's rule. Biological Journal of the Linnean Society 81:579-588.

Millen, V. 2006. Morphological evolution is accelerated among island mammals. PLoS Biology 4:e321.

Paradis, E., J. Claude, and K. Strimmer. 2004. APE: analyses of phylogenetics and evolution in R language. Bioinformatics 20:289290.

Raia, P., F. Carotenuto, and S. Meiri. 2010. One size does not fit all: no evidence for an optimal body size on islands. Global Ecology and Biogeography 19:475-484.

R Development Core Team. 2013. R: a language and environment for statistical computing. R Foundation for Statistical Computing, Vienna. http://www.R-project.org/.

Revell, L. 2011. Phylogenetic tools for comparative biology. http:// blog.phytools.org/2011/04/phylogenetic-rma-regression.html. Accessed September 25, 2015.

R Hackathon et al. 2014. phylobase: base package for phylogenetic structures and comparative data. R package, version 0.6.8. http:// CRAN.R-project.org $/$ package $=$ phylobase .

Rydell, J., and J. R. Speakman. 1995. Evolution of nocturnality in bats: potential competitors and predators during their early history. Biological Journal of the Linnean Society 54:183-191.

Sandom, C., S. Faurby, B. Sandel, and J.-C. Svenning. 2014. Global late Quaternary megafauna extinctions linked to humans, not climate change. Proceedings of the Roval Societv B: Biological Sciences 281:20133254.

Smith, F. A., A. G. Boyer, J. H. Brown, D. P. Costa, T. Dayan, S. K. M. Ernest, A. R. Evans, et al. 2010. The evolution of maximum body size of terrestrial mammals. Science 330:1216-1219.

Smith, F. A., S. K. Lyons, S. K. M. Ernest, K. E. Jones, D. M. Kaufman, T. Dayan, P. A. Marquet, J. H. Brown, and J. P. Haskell. 2003. Body mass of late Quaternary mammals. Ecology 84:3403.

Spiess, A. N. 2014. qpcR: modelling and analysis of real-time PCR data. R package, version 1.4-0. http://CRAN.R-project.org/package $=\mathrm{qpcR}$.

Stuenes, S. 1989. Taxonomy, habits, and relationships of the subfossil Madagascan hippopotami Hippopotamus lemerlei and H. madagascariensis. Iournal of Vertebrate Paleontology 9:241-268.

Thomas, J. A., J. J. Welch, R. Lanfear, and L. A. Bromham. 2010. Generation time effect on the rate of molecular evolution in invertebrates. Molecular Biology and Evolution 27:1173-1180.

Ting, N. 2008. Mitochondrial relationships and divergence dates of the African colobines: evidence of Miocene origins for the living colobus monkeys. Journal of Human Evolution 55:312-325. 
Turvey, S. T., and S. A. Fritz. 2011. The ghosts of mammals past: biological and geographical patterns of global mammalian extinction across the Holocene. Philosophical Transactions of the Roval Society B: Biological Sciences 366:2564-2576.

Uyeda, J. C., T. F. Hansen, S. J. Arnold, and J. Pienaar 2011. The millionyear wait for macroevolutionary bursts. Proceedings of the National Academy of Sciences of the USA 109:4191-4196.

Van Valen, L. 1973. Pattern and the balance of nature. Evolutionary Theory 1:31-49.

Welch, J. J. 2009. Testing the island rule: primates as a case study. Proceedings of the Roval Societv B: Biological Sciences 276:141-148.
Welch, J. J., O. R. P. Bininda-Emonds, and L. Bronham. 2008 Correlates of substitution rate variation in mammalian proteincoding sequences. BMC Evolutionary Biology 8:53-64.

Weyeneth, N., S. M. Goodman, and M. Ruedi. 2011. Do diversification models of Madagascar's biota explain the population structure of the endemic bat Myotis goudoti (Chiroptera: Vespertilionidae)? Iournal of Biogeography 38:44-54.

Associate Editor: Andrew J. Kerkhoff Editor: Susan Kalisz

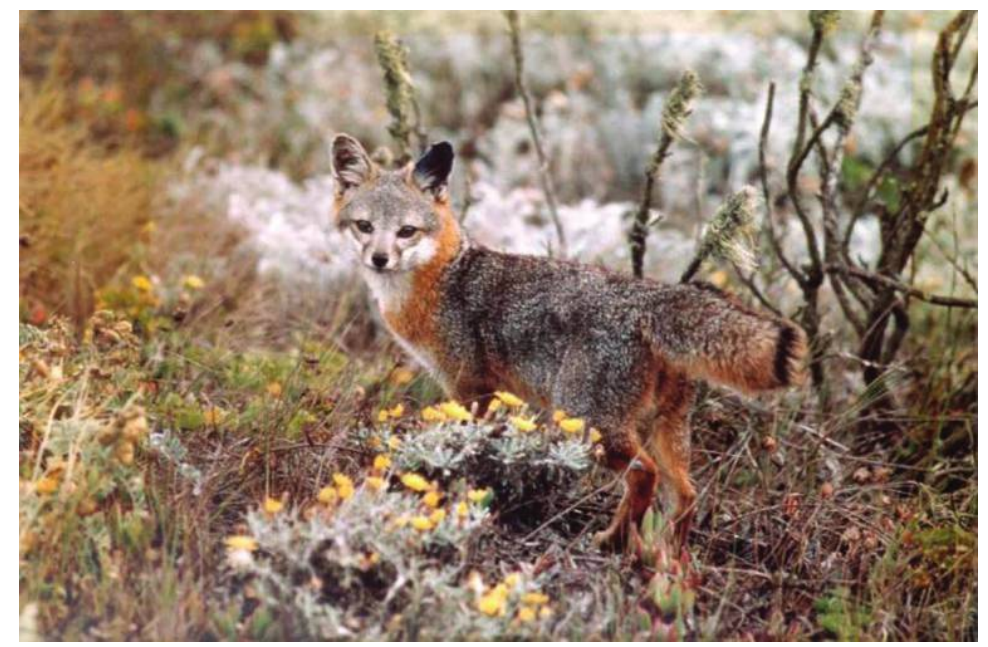

Island fox (Urocyon littoralis), a close relative and descendant of the widespread North American gray fox (Urocyon cinereoargenteus), endemic to the Californian Channel Islands. The island fox has a size of around $2 \mathrm{~kg}$, which is only about $50 \%$ of the size of its mainland ancestor. Like many other island species, the island fox has problems coexisting with nonnative predators, and in the 1990s, the species was highly endangered as a result of predation by golden eagles (Aquila chrysaetos), which in turn were able to colonize the islands because of human activity. The island fox, however, represents a rare case of conservation success, and extensive eagle management has meant that the species is no longer considered endangered. The fox used to share the islands with another dwarf, a very small mammoth species (Mammuthus exilis) with an estimated body size of $<200 \mathrm{~kg}$, but like the many other species of dwarf elephants, this is sadly extinct. Shown with permission from the photographer, Tim Coonan, US National Park Service. 